\title{
ROOTS OF GÅRDING HYPERBOLIC POLYNOMIALS
}

\author{
ARMIN RAINER
}

\begin{abstract}
We explore the regularity of the roots of Gårding hyperbolic polynomials and real stable polynomials. As an application we obtain new regularity results of Sobolev type for the eigenvalues of Hermitian matrices and for the singular values of arbitrary matrices. These results are optimal among all Sobolev spaces.
\end{abstract}

1. Introduction. A celebrated theorem of Bronshtein 9] (see also [26]) states that the roots of a monic univariate real-rooted polynomial of degree $d$ with coefficients $C^{d-1,1}$-functions defined in some open set $U \subseteq \mathbb{R}^{m}$ can be represented by functions that are locally Lipschitz in $U$. (A $C^{k, \alpha}$-function is by definition a function that is $k$-times continuously differentiable and whose partial derivatives of order $k$ satisfy a local $\alpha$-Hölder condition.) That means, if $a_{j} \in C^{d-1,1}(U), j=1, \ldots, d$, and all roots of the monic polynomial

$$
P_{a(x)}(Z)=Z^{d}+\sum_{j=1}^{d} a_{j}(x) Z^{d-j}
$$

are real for all $x \in U$, then there exist functions $\lambda_{j} \in C^{0,1}(U), j=1, \ldots, d$, such that

$$
P_{a(x)}(Z)=\prod_{j=1}^{d}\left(Z-\lambda_{j}(x)\right), \quad x \in U .
$$

Let us call any $d$-tuple of functions $\lambda=\left(\lambda_{1}, \ldots, \lambda_{d}\right)$ that satisfy (1.1) a system of the roots of $P_{a}$. One can show that any continuous system $\lambda=\left(\lambda_{1}, \ldots, \lambda_{d}\right)$ of the roots of $P_{a}$ with $a_{j} \in C^{d-1,1}(U), j=1, \ldots, d$, is locally Lipschitz. And there are explicit bounds for the $C^{0,1}$-norm of the $\lambda_{j}$ in terms of the coefficients $a_{j}$; see [26]. We shall refer to these results as Bronshtein's theorem.

That Bronshtein's theorem is sharp is manifest already in dimension $m=1$. If the coefficients $a=\left(a_{1}, \ldots, a_{d}\right)$ are of class $C^{d-1,1}$, then generally there are no differentiable systems of the roots of $P_{a}$; e.g. no function $\lambda$ with $\lambda^{2}=f$, where $f$ is the $C^{1,1}$-function $f(t)=t^{2} \sin ^{2}(\log |t|)$ for $t \neq 0$ and $f(0)=0$, is differentiable at 0 . On the other hand, if $a$ is of class $C^{d}$ (resp. $C^{2 d}$ ) and $m=1$, then there exists a $C^{1}$ (resp. twice differentiable) system of the roots of $P_{a}$; see [10] (and also [26]). However, there are non-negative $C^{\infty}$-functions $f$ on $\mathbb{R}$ such that no function $\lambda$ with $\lambda^{2}=f$ is of class $C^{1, \alpha}$ for any $\alpha>0$; cf. [6].

Date: April 21, 2021.

2010 Mathematics Subject Classification. 26C05, 15A18, 32A08, 46E35,

Key words and phrases. Hyperbolic polynomials, real stable polynomials, regularity of roots, Hermitian matrices, regularity of eigenvalues and singular values.

The author was supported by the Austrian Science Fund (FWF), START Programme Y963 and P 32905-N. 
There are various ways to generalize univariate real-rooted polynomials to multivariate polynomials. In this paper we will consider Gårding hyperbolic polynomials and real stable polynomials. These classes of polynomials are intimately related and have important applications in many different fields including PDEs, combinatorics, optimization, functional analysis, probability etc.

We will focus on the regularity of the roots of Gårding hyperbolic polynomials and real stable polynomials. By combining Bronshtein's theorem with the observation that the roots of Gårding hyperbolic polynomials are difference-convex functions we obtain uniform regularity results for the roots of the considered classes of real-rooted polynomials which in general are optimal among Sobolev spaces, see Theorem 4.6 .

As an application we obtain new regularity results for the eigenvalues of Hermitian matrices and for the singular values of arbitrary matrices. In particular, we will prove in Theorem 6.2 that any $C^{1,1}$-curve of Hermitian $d \times d$ matrices admits a system of its eigenvalues that is locally of Sobolev class $W^{2,1}$. This result is uniform, in the sense explained in Theorem 6.2(3), and optimal among all Sobolev spaces $W^{k, p}$, see Remark 6.3. (A $W^{k, p}$-function is by definition a $p$-integrable function with $p$-integrable weak partial derivatives up to order $k$.)

As a byproduct we obtain a new simple proof of the fact that a $C^{1}$-curve of Hermitian matrices admits a $C^{1}$-system of its eigenvalues. This was proved for symmetric matrices by [30] and for normal matrices by [28 by different methods.

Acknowledgment. This note arose from a discussion with Alexander Lytchak who introduced me to difference-convex functions.

2. Hyperbolic and real stable polynomials. We call a univariate polynomial $f(Z) \in \mathbb{C}[Z]$ real-rooted if all roots of $f$ are real; then also all coefficients are real and $f(Z) \in \mathbb{R}[Z]$. There is the following multivariate generalization.

Definition 2.1. A polynomial $f\left(Z_{1}, \ldots, Z_{n}\right) \in \mathbb{C}\left[Z_{1}, \ldots, Z_{n}\right]$ is said to be stable if $f\left(z_{1}, \ldots, z_{n}\right) \neq 0$ for all $\left(z_{1}, \ldots, z_{n}\right) \in \mathbb{C}^{n}$ with $\operatorname{Im}\left(z_{j}\right)>0$ for $j=1, \ldots, n$. A stable polynomial with real coefficients is called a real stable polynomial.

A univariate polynomial with real coefficients is real stable if and only if it is real-rooted. A polynomial $f\left(Z_{1}, \ldots, Z_{n}\right) \in \mathbb{C}\left[Z_{1}, \ldots, Z_{n}\right]$ is stable (resp. real stable) if and only if for all $x \in \mathbb{R}^{n}$ and all $v \in \mathbb{R}_{>0}^{n}$ the univariate polynomial $f(x+T v) \in \mathbb{C}[T]$ is stable (resp. real stable). Real stable polynomials played a crucial role in the recent proof of the Kadison-Singer conjecture 24.

A different but related multivariate generalization of real-rootedness is Gårding hyperbolicity.

Definition 2.2. A homogeneous polynomial $f\left(Z_{1}, \ldots, Z_{n}\right) \in \mathbb{R}\left[Z_{1}, \ldots, Z_{n}\right]$ is said to be Gårding hyperbolic with respect to a given vector $v \in \mathbb{R}^{n}$ if $f(v) \neq 0$ and for all $x \in \mathbb{R}^{n}$ the univariate polynomial $f(x-T v) \in \mathbb{R}[T]$ is real-rooted.

This notion was introduced by Gårding [12] in the 1950s. He showed that $f$ being hyperbolic with respect to a direction $v$ is a necessary and sufficient condition for local well-posedness of the Cauchy problem with principal symbol $f$ and initial data on a hyperplane with normal vector $v$. Hyperbolic polynomials have found many applications ever since, for instance, in Gurvits' proof [14] of the van der Waerden conjecture. 
Example 2.3. The determinant on the real vector space of $d \times d$ Hermitian matrices is Gårding hyperbolic with respect to the identity matrix II.

The relation between real stable and Gårding hyperbolic polynomials is captured in the following

Proposition 2.4 ([7, Proposition 1.1]). Let $f \in \mathbb{R}\left[Z_{1}, \ldots, Z_{n}\right]$ be of degree $d$ and let $f_{H} \in \mathbb{R}\left[Z_{1}, \ldots, Z_{n}, W\right]$ be the unique homogeneous polynomial of degree $d$ such that

$$
f_{H}\left(Z_{1}, \ldots, Z_{n}, 1\right)=f\left(Z_{1}, \ldots, Z_{n}\right) .
$$

Then $f$ is real stable if and only if $f_{H}$ is Garding hyperbolic with respect to every vector $v \in \mathbb{R}_{>0}^{n} \times\{0\}$.

Interesting examples of real stable polynomials (and hence of hyperbolic polynomials) are generated as follows (see [7. Proposition 1.12]): Let $A_{1}, \ldots, A_{n}$ be positive semidefinite $m \times m$ matrices and $B$ a (complex) Hermitian $m \times m$ matrix. Then the polynomial

$$
f\left(Z_{1}, \ldots, Z_{n}\right)=\operatorname{det}\left(\sum_{j=1}^{n} Z_{j} A_{j}+B\right)
$$

is either real stable or identically zero. Conversely, any real stable polynomial in two variables $Z_{1}$ and $Z_{2}$ can be written as $\pm \operatorname{det}\left(Z_{1} A_{1}+Z_{2} A_{2}+B\right)$, where $A_{j}$ are positive semidefinite and $B$ is symmetric [7, Theorem 1.13]. The latter result is based on (and corresponds to) the Lax conjecture [21] for Gårding hyperbolic polynomials which was recently established by [23] relying on work by [17, 34]; see also Remark 6.4

We refer to the survey article [5] for background on Gårding hyperbolic polynomials and more ways to generate examples.

3. Difference-convex functions. We shall work with the the class of differenceconvex functions. This is a subclass of the class of locally Lipschitz functions which arises as the smallest vector space that contains all continuous convex functions on a given set. We follow the survey article [4; see also [18].

Definition 3.1. Let $U \subseteq \mathbb{R}^{n}$ be a convex set. A function $f: U \rightarrow \mathbb{R}$ is said to be difference-convex $(D C)$ if it can be written as the difference of two continuous convex functions on $U$. A function $f$ is called locally difference-convex if each point in the domain of $f$ has a convex neighborhood on which $f$ is difference-convex.

Let us denote by $D C(U)$ the space of all difference-convex functions on $U$ and use the obvious notation $D C_{\text {loc }}(U)$ for the local class. We have $D C_{\text {loc }}(U) \subseteq C^{0,1}(U)$, by the local Lipschitz properties of locally bounded convex functions.

Next we collect some basic properties of difference-convex functions.

Lemma 3.2 (4]). Let $U \subseteq \mathbb{R}^{n}$ be an open convex set. Then:

(1) $D C(U)$ is an algebra.

(2) Each DC-function on $U$ is the difference of two non-negative convex continuous functions on $U$.

(3) If $f \in D C(U)$ then positive and negative parts $f^{ \pm}$of $f$ as well as $|f|$ belong to $D C(U)$.

For us the following properties will be crucial. 
Lemma 3.3 ([15]). We have:

(1) $f \in D C\left(\mathbb{R}^{n}\right)$ if and only if $f \in D C_{\mathrm{loc}}\left(\mathbb{R}^{n}\right)$.

(2) Let $A \subseteq \mathbb{R}^{m}$ be convex and either open or closed. Let $B \subseteq \mathbb{R}^{n}$ be convex and open. If $f: A \rightarrow B$ is $D C$ (that is each component is $D C$ ) and $g: B \rightarrow \mathbb{R}$ is $D C$, then $g \circ f$ is locally $D C$.

(3) Let $A \subseteq \mathbb{R}^{m}$ be convex and either open or closed. If a DC-function $f$ is nowhere vanishing on $A$, then $1 / f$ is $D C$ on $A$.

We shall also need

Lemma 3.4 (33. Lemma 4.8]). Let $U \subseteq \mathbb{R}^{n}$ be an open convex set and let $f_{j} \in$ $D C(U), j=1, \ldots, d$. If $f: U \rightarrow \mathbb{R}$ is continuous and $f(x) \in\left\{f_{1}(x), \ldots, f_{d}(x)\right\}$ for all $x \in U$, then $f \in D C(U)$.

Note that, if $U \subseteq \mathbb{R}^{n}$ is open, then $C^{1,1}(U) \subseteq D C_{\text {loc }}(U)$, cf. 32, Theorem 11] and [18, Section II]. In particular, all real polynomials on $\mathbb{R}^{n}$ belong to $D C\left(\mathbb{R}^{n}\right)$; see also [4, Section 3.3] for a direct argument.

On the other hand the first order partial derivatives of a difference-convex function $f: U \rightarrow \mathbb{R}$ have bounded variation, i.e., the weak second order partial derivatives of $f$ are signed Radon measures. This follows from Dudley's result 11] that a Schwartz distribution is a convex function if and only if its second derivative is a non-negative matrix-valued Radon measure. In one dimension, a real-valued function $f$ on a compact interval is difference-convex if and only if $f$ is absolutely continuous and $f^{\prime}$ has bounded variation.

4. Roots of Gårding hyperbolic polynomials. Let $f\left(Z_{1}, \ldots, Z_{n}\right) \in$ $\mathbb{R}\left[Z_{1}, \ldots, Z_{n}\right]$ be a homogeneous polynomial of degree $d$ which is Gårding hyperbolic with respect to a direction $v \in \mathbb{R}^{n}$. We may factorize

$$
f(x+T v)=f(v) \prod_{j=1}^{d}\left(T+\lambda_{j}^{\downarrow}(x)\right),
$$

where

$$
\lambda_{1}^{\downarrow}(x) \geq \ldots \geq \lambda_{d}^{\downarrow}(x)
$$

are the decreasingly ordered roots of $f(x-T v) \in \mathbb{R}[T]$. We call

$$
\lambda^{\downarrow}=\left(\lambda_{1}^{\downarrow}, \ldots, \lambda_{d}^{\downarrow}\right): \mathbb{R}^{n} \rightarrow \mathbb{R}^{d}
$$

the characteristic map of $f$ with respect to $v$ and $\lambda_{1}^{\downarrow}, \ldots, \lambda_{d}^{\downarrow}$ (in no particular order) the characteristic roots. The polynomial $f$ is recovered by $f(x)=f(v) \prod_{j=1}^{d} \lambda_{j}^{\downarrow}(x)$. We see that being Gårding hyperbolic with respect to a direction $v$ geometrically means that any affine line with direction $v$ meets the real hypersurface $\left\{x \in \mathbb{R}^{n}\right.$ : $f(x)=0\}$ in $d$ points (with multiplicities).

Note that for all $j=1, \ldots, d, r \in \mathbb{R}_{\geq 0}$, and $s \in \mathbb{R}$ we have

$$
\begin{aligned}
\lambda_{j}^{\downarrow}(r x+s v) & =r \lambda_{j}^{\downarrow}(x)+s, \quad \text { and } \\
\lambda_{j}^{\downarrow}(-x) & =-\lambda_{d+1-j}^{\downarrow}(x) .
\end{aligned}
$$

Indeed, $f(r x+s v+T v)=r^{d} f\left(x+r^{-1}(s+T) v\right)=r^{d} f(v) \prod_{j=1}^{d}\left(r^{-1}(s+T)+\lambda_{j}^{\downarrow}(x)\right)=$ $f(v) \prod_{j=1}^{d}\left(T+\left(s+r \lambda_{j}^{\downarrow}(x)\right)\right)$, by homogeneity. The case $r=0$ is even simpler: $f(T v)=T^{d} f(v)$. The second assertion can be seen analogously; the negative factor reverses the order of the roots. 
Gårding [13. Theorem 2] proved that $\lambda_{d}^{\downarrow}$ is concave which, by (4.11), is equivalent to $\lambda_{1}^{\downarrow}$ being convex. In view of (4.1) the largest root $\lambda_{1}^{\downarrow}$ is also positively homogeneous and thus sublinear. Recall that a function $\varphi$ on $\mathbb{R}^{n}$ is sublinear if $\varphi(r x+s y) \leq r \varphi(x)+s \varphi(y)$ for all $x, y \in \mathbb{R}^{n}$ and $r, s \geq 0$; it is easy to see that a positively homogeneous function $\varphi$ is convex if and only if it is sublinear. The connected component $C_{f}$ of the set $\mathbb{R}^{n} \backslash\{f=0\}$ which contains $v$ is an open convex cone, one has $C_{f}=\left\{x \in \mathbb{R}^{n}: \lambda_{d}^{\downarrow}(x)>0\right\}$, and $f$ is Gårding hyperbolic with respect to each $w \in C_{f}$.

In [5, Theorem 3.1] a method for generating Gårding hyperbolic polynomials from a given one was presented. Suppose that $f$ is a homogeneous polynomial of degree $d$ which is Gårding hyperbolic with respect to $v$ and has characteristic map $\lambda^{\downarrow}$. If $g$ is a homogeneous symmetric polynomial of degree $e$ on $\mathbb{R}^{d}$ which is Gårding hyperbolic with respect to $e_{1}+e_{2}+\cdots+e_{d}$ and has characteristic map $\mu^{\downarrow}$, then $g \circ \lambda^{\downarrow}$ is Gårding hyperbolic of degree $e$ with respect to $v$ and its characteristic map is $\mu^{\downarrow} \circ \lambda^{\downarrow}$. (Here and below $e_{i}$ denotes the $i$-th standard unit vector in $\mathbb{R}^{d}$.)

For instance, the homogeneous symmetric polynomial

$$
g_{k}\left(Y_{1}, \ldots, Y_{d}\right):=\prod_{\substack{I \subseteq\{1, \ldots, d\} \\|I|=k}} \sum_{i \in I} Y_{i} .
$$

of degree $\left(\begin{array}{l}d \\ k\end{array}\right)$ is Gårding hyperbolic with respect to $e_{1}+e_{2}+\cdots+e_{d}$ and has the characteristic roots $\mu_{I}(Y)=\frac{1}{k} \sum_{i \in I} Y_{i}$, where $I$ ranges over the subsets of $\{1, \ldots, d\}$ with $k$ elements. On the set $\left\{Y_{1} \geq Y_{2} \geq \cdots \geq Y_{d}\right\}$ we have $\mu_{I}(Y) \geq \mu_{J}(Y)$ if and only if $\sum_{i \in I} i \leq \sum_{j \in J} j$, in particular, $\mu_{\{1, \ldots, k\}}$ is the largest characteristic root. Then $g_{k} \circ \lambda^{\downarrow}$ is Gårding hyperbolic with respect to $v$ and has the largest characteristic root $\mu_{\{1, \ldots, k\}} \circ \lambda^{\downarrow}=\frac{1}{k} \sum_{i=1}^{k} \lambda_{i}^{\downarrow}$. From Gårding's result we may conclude that, for all $k=1, \ldots, d$, the sum of the $k$ largest roots

$$
\sigma_{k}:=\sum_{i=1}^{k} \lambda_{i}^{\downarrow}
$$

is a sublinear function on $\mathbb{R}^{n}$. We immediately obtain

Proposition 4.1. Let $f\left(Z_{1}, \ldots, Z_{n}\right) \in \mathbb{R}\left[Z_{1}, \ldots, Z_{n}\right]$ be a homogeneous polynomial of degree $d$ which is Gairding hyperbolic with respect to a direction $v \in \mathbb{R}^{n}$. The characteristic map $\lambda^{\downarrow}: \mathbb{R}^{n} \rightarrow \mathbb{R}^{d}$ is globally Lipschitz and difference-convex on $\mathbb{R}^{n}$.

Proof. Any finite sublinear function on $\mathbb{R}^{n}$ is globally Lipschitz; this follows from [31, Corollary 10.5.1]. By the above discussion, the functions $\sigma_{k}, k=1, \ldots, d$, are convex and globally Lipschitz. Thus each $\lambda_{k}^{\downarrow}=\sigma_{k}-\sigma_{k-1}$ is difference-convex and globally Lipschitz.

Combined with Lemma 3.4 this leads to

Corollary 4.2. In the setting of Proposition 4.1 every continuous function $\lambda$ : $\mathbb{R}^{n} \rightarrow \mathbb{R}$ satisfying $f(x-\lambda(x) v)=0$ for all $x \in \mathbb{R}^{n}$ is globally Lipschitz and difference-convex on $\mathbb{R}^{n}$.

Proof. That $\lambda \in D C\left(\mathbb{R}^{n}\right)$ is an immediate consequence of Lemma 3.4 and Proposition 4.1. For arbitrary $x, y \in \mathbb{R}^{n}$ consider the set $[x, y]:=\{(1-t) x+t y: t \in[0,1]\}$ equipped with the linear order induced by $[0,1]$. By assumption, there is a finite 
sequence of points $x=: x_{0}<x_{1}<\cdots<x_{k}:=y$ with $k \leq d$ such that for each $i=1, \ldots, k$ there exists $j_{i}$ such that $\lambda\left(x_{i-1}\right)=\lambda_{j_{i}}^{\downarrow}\left(x_{i-1}\right)$ and $\lambda\left(x_{i}\right)=\lambda_{j_{i}}^{\downarrow}\left(x_{i}\right)$. Then

$$
\frac{|\lambda(y)-\lambda(x)|}{|y-x|} \leq \sum_{i=1}^{k} \frac{\left|\lambda\left(x_{i}\right)-\lambda\left(x_{i-1}\right)\right|}{\left|x_{i}-x_{i-1}\right|} \cdot \frac{\left|x_{i}-x_{i-1}\right|}{|y-x|} \leq \sum_{i=1}^{k} \frac{\left|\lambda_{j_{i}}^{\downarrow}\left(x_{i}\right)-\lambda_{j_{i}}^{\downarrow}\left(x_{i-1}\right)\right|}{\left|x_{i}-x_{i-1}\right|}
$$

which implies that $\lambda$ is globally Lipschitz, by Proposition 4.1

Let $\operatorname{Hyp}_{n}^{d}(v)$ denote the space of all homogeneous polynomials $f\left(Z_{1}, \ldots, Z_{n}\right) \in$ $\mathbb{R}\left[Z_{1}, \ldots, Z_{n}\right]$ of degree $d$ which are Gårding hyperbolic with respect to a fixed vector $v \in \mathbb{R}^{n}$. By identifying $f \in \operatorname{Hyp}_{n}^{d}(v)$ with the sequence of its coefficients (with respect to a fixed order of the monomials) we view $\operatorname{Hyp}_{n}^{d}(v)$ as a subset of $\mathbb{R}^{N}$ where $N=\left(\begin{array}{c}n+d-1 \\ d\end{array}\right)$. Then the subset $\operatorname{Hyp}_{n}^{\circ}(v)$, consisting of all those $f \in \operatorname{Hyp}_{n}^{d}(v)$ for which the roots of $f(x-T v)$ are simple for every $x$ not proportional to $v$, is open. And $\operatorname{Hyp}_{n}^{d}(v)$ is the part of the closure of $\operatorname{Hyp}_{n}^{\circ}(v)$ where $f(v) \neq 0$. See [25] in which also the connectivity properties of these spaces are discussed.

By a $C^{k, \alpha}$-mapping $f: \mathbb{R}^{m} \rightarrow \operatorname{Hyp}_{n}^{d}(v)$ we mean a mapping with values in $\operatorname{Hyp}_{n}^{d}(v)$ which is of class $C^{k, \alpha}$ when considered as mapping to $\mathbb{R}^{N}$. For each $y \in \mathbb{R}^{m}$ we have the characteristic map $\lambda^{\downarrow}(y)(\cdot)$ of $f(y)$. By slight abuse of notation we write

$$
\lambda^{\downarrow}: \mathbb{R}^{m} \times \mathbb{R}^{n} \rightarrow \mathbb{R}^{d}
$$

and call it the characteristic map of $f$ with respect to $v$. Its regularity properties are stated in the following

Proposition 4.3. If $f: \mathbb{R}^{m} \rightarrow \operatorname{Hyp}_{n}^{d}(v)$ is a mapping of class $C^{d-1,1}$, then its characteristic map $\lambda^{\downarrow}: \mathbb{R}^{m} \times \mathbb{R}^{n} \rightarrow \mathbb{R}^{d}$ is locally Lipschitz.

Proof. Bronshtein's theorem [9] (see also [26]) implies that the $\operatorname{roots} \lambda_{j}^{\downarrow}(y, x), j=$ $1, \ldots, d$ of the univariate real-rooted polynomial

$$
\mathbb{R}^{m} \times \mathbb{R}^{n} \ni(y, x) \mapsto f(y)(x-T v) \in \mathbb{R}[T]
$$

are locally Lipschitz in $(y, x)$.

Note that in Proposition 4.3 we need the assumption that the coefficients of $f$ are of class $C^{d-1,1}$, since the assumptions of Bronshtein's theorem are sharp.

By Proposition 4.1 the behavior of the characteristic map $\lambda^{\downarrow}: \mathbb{R}^{m} \times \mathbb{R}^{n} \rightarrow$ $\mathbb{R}^{d},(y, x) \mapsto \lambda^{\downarrow}(y, x)$ is essentially better in the $x$ variables. This will be further investigated in the next theorem, that is Theorem 4.6. To this end we need the following

Lemma 4.4. Let $\lambda=\left(\lambda_{1}, \ldots, \lambda_{d}\right): \mathbb{R} \rightarrow \mathbb{R}^{d}$ be of class $C^{1}$ and assume that the coefficients of the polynomial $P(t)(Z)=\prod_{j=1}^{d}\left(Z-\lambda_{j}(t)\right)$ are of class $W_{\mathrm{loc}}^{2,1}$. Then each derivative $\lambda_{j}^{\prime}, j=1, \ldots, d$, has the Luzin $(N)$ property (i.e. sets of Lebesgue measure zero are mapped to sets of Lebesgue measure zero).

Proof. Note that it is enough to show the Luzin (N) property on bounded intervals, since $\mathbb{R}$ can be covered by countably many of them. We proceed by induction on $d$. The case $d=1$ is clear, since in this case the derivative of the only root is locally absolutely continuous, by assumption. Let $d>1$.

Let $A$ denote the closed set of all $t \in \mathbb{R}$ such that $\lambda_{1}(t)=\cdots=\lambda_{d}(t)$. On an open neighborhood $I$ of each point $t_{0} \in A^{c}:=\mathbb{R} \backslash A$ we have a splitting $P(t)=P_{1}(t) P_{2}(t)$ 
into polynomials with positive degree and $W_{\text {loc }}^{2,1}$ coefficients, by the inverse function theorem (cf. [26, Lemma 3.2]); here we use that the superposition with a real analytic function preserves the class $W_{\mathrm{loc}}^{2,1}$, cf. [8, Theorem 2]. On $I$ we have a partition of $\left\{\lambda_{1}, \ldots, \lambda_{d}\right\}$ into the roots of $P_{1}$ and the roots of $P_{2}$. By induction hypothesis, for each $j$ the restriction $\left.\lambda_{j}^{\prime}\right|_{I}$ has the Luzin $(\mathrm{N})$ property. Since $A^{c}$ can be covered by countably many open neighborhoods of type $I$, we see that each $\left.\lambda_{j}^{\prime}\right|_{A^{c}}$ has the Luzin $(\mathrm{N})$ property.

Let $A^{\prime}$ denote the set of accumulation points of $A$. Let $a_{1}(t)$ denote the coefficient of $Z^{d-1}$ in $P(t)$. For $t \in A$ we have

$$
a_{1}(t)=-\lambda_{1}(t)-\cdots-\lambda_{d}(t)=-d \cdot \lambda_{j}(t), \quad \text { for all } j .
$$

And hence for $t \in A^{\prime}$ and all $j$ we have

$$
\lambda_{j}^{\prime}(t)=\lim _{A \ni t_{n} \rightarrow t} \frac{\lambda_{j}\left(t_{n}\right)-\lambda_{j}(t)}{t_{n}-t}=-\frac{1}{d} \lim _{A \ni t_{n} \rightarrow t} \frac{a_{1}\left(t_{n}\right)-a_{1}(t)}{t_{n}-t}=-\frac{a_{1}^{\prime}(t)}{d} .
$$

By assumption $a_{1}^{\prime}$ is absolutely continuous and hence has the Luzin (N) property. If $E \subseteq \mathbb{R}$ is a set of Lebesgue measure zero, then also

$$
\begin{aligned}
\lambda_{j}^{\prime}(E) & =\lambda_{j}^{\prime}\left(E \cap A^{c}\right) \cup \lambda_{j}^{\prime}\left(E \cap A^{\prime}\right) \cup \lambda_{j}^{\prime}\left(E \cap A \backslash A^{\prime}\right) \\
& =\lambda_{j}^{\prime}\left(E \cap A^{c}\right) \cup\left(-\frac{1}{d}\right) a_{1}^{\prime}\left(E \cap A^{\prime}\right) \cup \lambda_{j}^{\prime}\left(E \cap A \backslash A^{\prime}\right)
\end{aligned}
$$

has Lebesgue measure zero, noting that $A \backslash A^{\prime}$ is discrete and thus countable.

Let $f \in \operatorname{Hyp}_{n}^{d}(v)$ have characteristic map $\lambda^{\downarrow}: \mathbb{R}^{n} \rightarrow \mathbb{R}^{d}$. Let $x: \mathbb{R} \rightarrow \mathbb{R}^{n}$ be a curve. A mapping $\lambda=\left(\lambda_{1}, \ldots, \lambda_{d}\right): \mathbb{R} \rightarrow \mathbb{R}^{d}$ is called a system of the roots of $f$ along $x$ if $\lambda(t)$ and $\lambda^{\downarrow}(x(t))$ coincide as unordered $d$-tuples for all $t$.

Lemma 4.5. Let $f \in \operatorname{Hyp}_{n}^{d}(v)$ and let $x: \mathbb{R} \rightarrow \mathbb{R}^{n}$ be a $C^{1}$-curve. Then there exists a differentiable system $\lambda=\left(\lambda_{1}, \ldots, \lambda_{d}\right)$ of the roots of $f$ along $x$.

Proof. Let $\lambda_{0}$ be a $p$-fold root of $f\left(x\left(t_{0}\right)-T v\right)$. We claim that the polynomial $f(x(t)-T v)$ has $p$ roots of the form $\lambda_{0}+\left(t-t_{0}\right) \mu_{j}(t)$, where the $\mu_{j}$ are functions which are defined near $t_{0}$ and continuous at $t_{0}$.

We may assume without loss of generality that $t_{0}=0$. In view of (4.1) we may also assume that $\lambda_{0}=0$. We consider the localization $f_{x(0)}$ of $f$ at $x(0)$ defined by

$$
t^{d} f\left(t^{-1} x(0)+\xi\right)=f(x(0)+t \xi)=t^{p} f_{x(0)}(\xi)+O\left(t^{p+1}\right),
$$

where $f_{x(0)}(\xi)$ is the first coefficient in the above expansion which does not vanish identically. Since

$$
f(x(0)+t v)=f(v) \prod_{j=1}^{d}\left(t+\lambda_{j}^{\downarrow}(x(0))\right)=t^{p} f(v) \prod_{\lambda_{j}^{\downarrow}(x(0)) \neq \lambda_{0}}\left(t+\lambda_{j}^{\downarrow}(x(0))\right),
$$

the exponent $p$ in (4.3) is precisely the multiplicity of $\lambda_{0}$. The homogeneous polynomial $f_{x(0)}$ has degree $p$ and is Garding hyperbolic with respect to $v$; see $\underline{3}$, Lemma $3.42]$.

Since $x$ is of class $C^{1}$, we have $x(t)=x(0)+t \tilde{x}(t)$ for a continuous function $\tilde{x}$. We are interested in the solutions of the equation

$$
f(x(0)+t \tilde{x}(t)-t Y v)=0
$$

By (4.3) we may equivalently study the equation

$$
f_{x(0)}(\tilde{x}(t)-Y v)+O(t)=0 .
$$


This is a polynomial equation in the unknown $Y$ with coefficients which depend continuously on $t$ in a neighborhood of 0 . It follows that there exist $p$ solutions $\mu_{1}(t), \ldots, \mu_{p}(t)$ (since $p$ is the degree of $f_{x(0)}$ ) for $t$ near 0 which are continuous at $t=0$. This implies the claim.

Let us indicate how to construct a global differentiable system $\lambda=\left(\lambda_{1}, \ldots, \lambda_{d}\right)$ of the roots of $f$ along $x$ from the local data provided by the claim; we follow the argument in [1, 4.3]. We start with the continuous system $\nu_{1} \geq \nu_{2} \geq \cdots \geq \nu_{d}$, where $\nu_{j}:=\lambda_{j}^{\downarrow} \circ x$. Fix $i \neq j$ and $t_{0} \in \mathbb{R}$. If $\nu_{i}\left(t_{0}\right)=\nu_{j}\left(t_{0}\right)$, then, by the claim, $\nu_{i}(t)-\nu_{j}(t)=\left(t-t_{0}\right) \nu_{i j}(t)$ near $t_{0}$, where $\nu_{i j}$ is a function which is continuous at $t_{0}$. Let $E_{i j}$ be the closed set of $t_{0} \in \mathbb{R}$ such that $\nu_{i}\left(t_{0}\right)=\nu_{j}\left(t_{0}\right)$ and $\nu_{i j}\left(t_{0}\right)=0$. At the points of $E_{i j}$ the roots $\nu_{i}$ and $\nu_{j}$ are differentiable and the derivatives $\nu_{i}^{\prime}$ and $\nu_{j}^{\prime}$ coincide. Let us define

$$
\lambda_{k}(t):=\nu_{\sigma_{t}(k)}(t), \quad k=1, \ldots, d,
$$

where $\sigma_{t}$ is the permutation

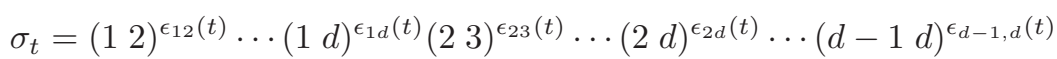

and $\epsilon_{i j}(t) \in\{0,1\}$ are determined as follows. In each connected component $I$ of $\mathbb{R} \backslash E_{i j}$ choose a point $t_{I}$ and set $\epsilon_{i j}\left(t_{I}\right)=0$. Going left and right from $t_{I}$ change $\epsilon_{i j}$ in each point $t_{0} \in I$, where $\nu_{i}\left(t_{0}\right)=\nu_{j}\left(t_{0}\right)$ and $\nu_{i j}\left(t_{0}\right) \neq 0$ (including $t_{I}$ if it is such a point). These points accumulate only in $E_{i j}$, where any choice for $\epsilon_{i j}$ is good. It follows that $\lambda=\left(\lambda_{1}, \ldots, \lambda_{d}\right)$ is a global differentiable system of the roots of $f$ along $x$.

We are ready to state and prove the theorem.

Theorem 4.6. Let $f \in \operatorname{Hyp}_{n}^{d}(v)$. Let $x \in D C\left(\mathbb{R}, \mathbb{R}^{n}\right) \cap W_{\mathrm{loc}}^{2,1}\left(\mathbb{R}, \mathbb{R}^{n}\right)$; this is, for instance, fulfilled if $x \in C^{1,1}\left(\mathbb{R}, \mathbb{R}^{n}\right)$.

(1) Any differentiable system $\lambda=\left(\lambda_{1}, \ldots, \lambda_{d}\right)$ of the roots of $f$ along $x$ is actually of class

$$
\lambda \in C^{1}\left(\mathbb{R}, \mathbb{R}^{d}\right) \cap D C\left(\mathbb{R}, \mathbb{R}^{d}\right) \cap W_{\mathrm{loc}}^{2,1}\left(\mathbb{R}, \mathbb{R}^{d}\right) .
$$

(2) If $x: \mathbb{R} \rightarrow \mathbb{R}^{n}$ is of class $C^{1}$, then there exists a differentiable (thus $C^{1}$ ) system $\lambda=\left(\lambda_{1}, \ldots, \lambda_{d}\right)$ of the roots of $f$ along $x$.

(3) The result is uniform in the following sense: Let $I \subseteq \mathbb{R}$ be a bounded open interval. Let $U$ be an open neighborhood of the closure of $I^{1+k}$ in $\mathbb{R}^{1+k}$. Suppose that $x: U \rightarrow \mathbb{R}^{n}$ is such that

- $x$ is locally $D C$ on $U$,

- $x(\cdot, r) \in C^{1}\left(\bar{I}, \mathbb{R}^{n}\right) \cap W^{2,1}\left(I, \mathbb{R}^{n}\right)$ for all $r \in \bar{I}^{k}$.

Assume that, for each $r \in \bar{I}^{k}$, a $C^{1}$-system $\lambda(\cdot, r)$ of the roots of $f$ along $x(\cdot, r)$ is fixed. Then the family $\lambda(\cdot, r)$, for $r \in \bar{I}^{k}$, is bounded in $C^{1}\left(\bar{I}, \mathbb{R}^{d}\right)$ and there is a non-negative $L^{1}$-function $m: I^{k} \rightarrow \mathbb{R}_{\geq 0}$ such that

$$
\|\lambda(\cdot, r)\|_{W^{2,1}\left(I, \mathbb{R}^{d}\right)} \leq m(r), \quad \text { for a.e. } r \in I^{k} .
$$

Proof. (1) Let $\lambda=\left(\lambda_{1}, \ldots, \lambda_{d}\right)$ be a differentiable system of the roots of $f$ along $x$. By Lemma 3.3. $\lambda^{\downarrow} \circ x$ is $D C$ on $\mathbb{R}$. Then $\lambda$ is $D C$ on $\mathbb{R}$, by Corollary 4.2 A differentiable $D C$-function $\varphi$ on $\mathbb{R}$ is of class $C^{1}$ and the difference of two convex $C^{1}$-functions (see [18, Theorem 2.1]); indeed, by Darboux's theorem, $\varphi^{\prime}$ has the 
intermediate value property and so $\varphi^{\prime}$ having (locally) bounded variation implies that $\varphi^{\prime}$ is continuous. It follows that $\lambda$ is of class $C^{1}$.

In order to conclude that $\lambda \in W_{\text {loc }}^{2,1}\left(\mathbb{R}, \mathbb{R}^{n}\right)$ it suffices to show that each $\lambda_{j}^{\prime}$ has the Luzin $(\mathrm{N})$ property which follows from Lemma 4.4 in fact a continuous function of pointwise bounded variation with the Luzin $(\mathrm{N})$ property is absolutely continuous, cf. [22, Corollary 3.27].

(2) This is Lemma 4.5

(3) The Lipschitz constant of $\left(\lambda^{\downarrow} \circ x\right)(\cdot, r)$ on $\bar{I}$ is uniformly bounded in $r \in \bar{I}^{k}$ by the chain rule, since $\lambda^{\downarrow}$ is Lipschitz, by Proposition 4.1 By an argument similar to the one in the proof of Corollary 4.2 we may infer the boundedness of the Lipschitz constant of $\lambda(\cdot, r)$ on $\bar{I}$. It follows that the family $\lambda(\cdot, r), r \in \bar{I}^{k}$, is bounded in $C^{1}\left(\bar{I}, \mathbb{R}^{d}\right)$.

By Lemma 3.3. $\lambda^{\downarrow} \circ x$ is locally $D C$ on $U$. In particular, the directional variation $V_{e_{1}}\left(\partial_{1}\left(\lambda^{\downarrow} \circ x\right), I^{1+k}\right)$ of $\partial_{1}\left(\lambda^{\downarrow} \circ x\right)$ on $I^{1+k}$ is finite, where $e_{1}$ denotes the first standard unit vector. We have, see e.g [2, (3.100)],

$$
V_{e_{1}}\left(\partial_{1}\left(\lambda^{\downarrow} \circ x\right), I^{1+k}\right)=\int_{I^{k}} V\left(\partial_{1}\left(\lambda^{\downarrow} \circ x\right)(\cdot, r), I\right) d r,
$$

where $V\left(\partial_{1}\left(\lambda^{\downarrow} \circ x\right)(\cdot, r), I\right)$ is the variation of $\partial_{1}\left(\lambda^{\downarrow} \circ x\right)(\cdot, r)$ on $I$. The variation of $\partial_{1}\left(\lambda^{\downarrow} \circ x\right)(\cdot, r)$ on $I$ dominates the variation of $\partial_{1} \lambda(\cdot, r)$ on $I$ (up to a constant which depends only on $d$ ), since for each $j=1, \ldots, d$ and a.e. $t$ we have

$$
\partial_{1} \lambda_{j}(t, r) \in\left\{\partial_{1}\left(\lambda_{1}^{\downarrow} \circ x\right)(t, r), \ldots, \partial_{1}\left(\lambda_{d}^{\downarrow} \circ x\right)(t, r)\right\},
$$

and $\partial_{1} \lambda_{j}(t, r)$ is continuous in $t$. Since $\partial_{1} \lambda_{j}(\cdot, r)$ is absolutely continuous on $I$, by (1), its $W^{1,1}$-norm coincides with its $B V$-norm on $I$. Together with the boundedness in $C^{1}\left(\bar{I}, \mathbb{R}^{d}\right)$ the assertion (4.5) follows easily.

Remark 4.7. If the system $\lambda=\left(\lambda_{1}, \ldots, \lambda_{d}\right)$ of the roots of $f$ along $x$ is not differentiable at $t_{0}$, then $\lambda$ is not of class $W^{2,1}$ in any neighborhood of $t_{0}$.

In several variables the characteristic roots do not admit differentiable rearrangements. But for real analytic families of Gårding hyperbolic polynomials the characteristic roots can be chosen real analytically locally after blowing up the parameter space.

Proposition 4.8. If $f: \mathbb{R}^{m} \rightarrow \operatorname{Hyp}_{n}^{d}(v)$ is real analytic and $x: \mathbb{R}^{\ell} \rightarrow \mathbb{R}^{n}$ is real analytic, then there exists a locally finite composite of blowing-ups with smooth centers $\tau=\left(\tau_{1}, \tau_{2}\right): Y \rightarrow \mathbb{R}^{m} \times \mathbb{R}^{\ell}$ such that for each $y_{0} \in Y$ there is a neighborhood $U$ and a real analytic system of the roots of the univariate polynomial

$$
U \ni y \mapsto f\left(\tau_{1}(y)\right)\left(x\left(\tau_{2}(y)\right)-T v\right) \in \mathbb{R}[T] .
$$

If $f: \mathbb{R} \rightarrow \operatorname{Hyp}_{n}^{d}(v)$ is real analytic and $x: \mathbb{R} \rightarrow \mathbb{R}^{n}$ is a real analytic curve, then there exists a global real analytic system of the roots of

$$
\mathbb{R} \ni s \mapsto f(s)(x(s)-T v) \in \mathbb{R}[T] .
$$

Proof. The statement follows from [20, Theorem 5.8] applied to the real-rooted polynomial (4.2). The (one-dimensional) supplement follows from Rellich's theorem [29]; see also [1, Theorem 5.1].

Remark 4.9. Analogous results follow for suitable quasianalytic classes thanks to a corresponding theorem for real-rooted polynomials, see [27]. 
Remark 4.10. It is shown in [16, Theorem 4.2] that for any $f \in \operatorname{Hyp}_{n}^{d}(v), x_{0} \in \mathbb{R}^{n}$, and $w \in C_{f}$ there is a system $\lambda=\left(\lambda_{1}, \ldots, \lambda_{d}\right): \mathbb{R} \rightarrow \mathbb{R}^{d}$ of the roots of $f$ along $t \mapsto x_{0}+t w$ which is real analytic and such that each $\lambda_{j}: \mathbb{R} \rightarrow \mathbb{R}$ is strictly increasing and surjective. The inverses of the $\lambda_{j}$ form a system of the characteristic roots of $f \in \operatorname{Hyp}_{n}^{d}(w)$ along $t \mapsto x_{0}+t v$.

5. Roots of real stable polynomials. The regularity results for Gårding hyperbolic polynomials of the previous section immediately give regularity results for real stable polynomials thanks to the connection presented in Proposition 2.4. Let us denote by RStab ${ }_{n}^{d}$ the set of real stable polynomials of degree $d$ in $n$ variables. Then Proposition 2.4 defines a bijection

$$
\operatorname{RStab}_{n}^{d} \rightarrow \bigcap_{v \in \mathbb{R}_{>0}^{n} \times\{0\}} \operatorname{Hyp}_{n}^{d}(v), \quad f \mapsto f_{H} .
$$

Suppose that $f \in \operatorname{RStab}_{n}^{d}$. For all $v \in \mathbb{R}_{>0}^{n} \times\{0\}$ we may consider the characteristic map $\lambda_{v}^{\downarrow}: \mathbb{R}^{n+1} \rightarrow \mathbb{R}^{d}$ of $f_{H}$ with respect to $v$. Then the mapping $\left.\lambda_{v}^{\downarrow}\right|_{\left\{x_{n+1}=1\right\}}$ is globally Lipschitz and difference-convex and its components represent the roots of $f\left(x^{\prime}-T v^{\prime}\right) \in \mathbb{R}[T]$, where $x^{\prime}=\left(x_{1}, \ldots, x_{n}\right)$ and $v^{\prime}=\left(v_{1}, \ldots, v_{n}\right)$. It is then straightforward to transfer the regularity results for Gårding hyperbolic polynomials of the previous section.

Finally, note that we can also vary the direction $v$ : if $f: \mathbb{R}^{m} \rightarrow \operatorname{RStab}_{n}^{d}$ and $v: \mathbb{R}^{\ell} \rightarrow \mathbb{R}_{>0}^{n}$ are $C^{d-1,1}$-mappings, then the roots of the univariate real-rooted polynomial

$$
\mathbb{R}^{m} \times \mathbb{R}^{\ell} \times \mathbb{R}^{n} \ni(s, t, x) \mapsto f(s)(x-T v(t)) \in \mathbb{R}[T]
$$

are locally Lipschitz in $(s, t, x)$, by Bronshtein's theorem [9] (see also [26]).

6. Eigenvalues of Hermitian matrices. Let $\operatorname{Herm}(d)$ denote the real vector space of complex $d \times d$ Hermitian matrices. The determinant det is Gårding hyperbolic with respect to the identity matrix $\mathbb{I} \in \operatorname{Herm}(d)$. The characteristic map $\lambda^{\downarrow}$ of det with respect to $\mathbb{I}$ assigns to a Hermitian matrix $A \in \operatorname{Herm}(d)$ its eigenvalues in decreasing order

$$
\lambda_{1}^{\downarrow}(A) \geq \ldots \geq \lambda_{d}^{\downarrow}(A) .
$$

As a direct consequence of Proposition 4.1 and the discussion preceding it we get

Corollary 6.1. The characteristic map $\lambda^{\downarrow}: \operatorname{Herm}(d) \rightarrow \mathbb{R}^{d}$ is globally Lipschitz and difference-convex on $\operatorname{Herm}(d)$. The sum $\sum_{i=1}^{k} \lambda_{i}^{\downarrow}$, for $k=1, \ldots, d$, of the $k$ largest eigenvalues is sublinear.

The following result is a special case of Theorem 4.6.

Theorem 6.2. Let $A: \mathbb{R} \rightarrow \operatorname{Herm}(d)$ be a curve of $d \times d$ Hermitian matrices of class $D C \cap W_{\text {loc }}^{2,1}$ on $\mathbb{R}$.

(1) Any differentiable system $\lambda=\left(\lambda_{1}, \ldots, \lambda_{d}\right)$ of the eigenvalues of $A$ is actually of class

$$
\lambda \in C^{1}\left(\mathbb{R}, \mathbb{R}^{d}\right) \cap D C\left(\mathbb{R}, \mathbb{R}^{d}\right) \cap W_{\text {loc }}^{2,1}\left(\mathbb{R}, \mathbb{R}^{d}\right) .
$$

(2) If $A$ additionally is of class $C^{1}$, then there exists a differentiable (thus $C^{1}$ ) system $\lambda=\left(\lambda_{1}, \ldots, \lambda_{d}\right)$ of the eigenvalues of $A$.

(3) The result is uniform in the following sense: Let $I \subseteq \mathbb{R}$ be a bounded open interval. Let $U$ be an open neighborhood of the closure of $I^{1+k}$ in $\mathbb{R}^{1+k}$. Suppose that $A: U \rightarrow \operatorname{Herm}(d)$ is such that 
- A is locally $D C$ on $U$,

- $A(\cdot, r)$ is of class $C^{1} \cap W^{2,1}$ on $\bar{I}$ for all $r \in \bar{I}^{k}$.

Assume that, for each $r \in \bar{I}^{k}$, a $C^{1}$-system $\lambda(\cdot, r)$ of the eigenvalues of $A(\cdot, r)$ is fixed. Then the family $\lambda(\cdot, r)$, for $r \in \bar{I}^{k}$, is bounded in $C^{1}\left(\bar{I}, \mathbb{R}^{d}\right)$ and there is a non-negative $L^{1}$-function $m: I^{k} \rightarrow \mathbb{R}_{\geq 0}$ such that

$$
\|\lambda(\cdot, r)\|_{W^{2,1}\left(I, \mathbb{R}^{d}\right)} \leq m(r), \quad \text { for a.e. } r \in I^{k} .
$$

Note that the assumptions on $A$ are in particular satisfied if $A$ is of class $C^{1,1}$.

Remark 6.3. The conclusion of this theorem is best-possible among all Sobolev spaces $W^{k, p}$. Indeed, by the Sobolev inequality, $W^{k, p}$-regularity with $k+p>2$ would imply $C^{1, \alpha}$-regularity with some $\alpha>0$, contradicting the counter-example of [19: there is a $C^{\infty}$-curve of symmetric $2 \times 2$ matrices the eigenvalues of which do not admit a $C^{1, \alpha}$-system for any $\alpha>0$.

Remark 6.4. Statement (2) in Theorem 6.2] is due to Rellich [30] in the case of symmetric matrices, and it was proved for normal matrices in 28]. Lemma 4.5 gives an independent proof for Hermitian matrices.

Conversely, (2) in Theorem 6.2 implies Lemma 4.5 if $n \leq 3$ : The Lax conjecture (posed 1958 in 21]) which by now is a theorem (as 23 discovered the conjecture follows from a theorem of [17, 34]) states that a homogeneous polynomial $f$ on $\mathbb{R}^{3}$ is Gårding hyperbolic of degree $d$ with respect to the direction $e_{1}$ with $f\left(e_{1}\right)=1$ if and only if there exist real symmetric $d \times d$ matrices $A$ and $B$ such that

$$
f(x, y, z)=\operatorname{det}(x \mathbb{I}+y A+z B) .
$$

So, for $n \leq 3$, Theorem 4.6 follows from Theorem 6.2. A representation of type (6.1) is in general not possible for Gårding hyperbolic polynomials on $\mathbb{R}^{n}$ with $n \geq 4$. Indeed, the dimension of $\operatorname{Hyp}_{n}^{d}(v)$ is $\left(\begin{array}{c}n+d-1 \\ d\end{array}\right)$ while the set of polynomials in $\mathbb{R}\left[X_{1}, \ldots, X_{n}\right]$ of the form

$$
\operatorname{det}\left(X_{1} \mathbb{I}+X_{2} A_{2}+\cdots+X_{n} A_{n}\right),
$$

where $A_{i}$ are real symmetric $d \times d$ matrices, is at most $(n-1) \cdot\left(\begin{array}{c}d+1 \\ 2\end{array}\right)$.

A particular homogeneous polynomial of degree 2 which is Gårding hyperbolic with respect to $e_{1}$ but cannot be represented in the form (6.2) is the Lorentzian polynomial $f(X)=X_{1}^{2}-X_{2}^{2}-\cdots-X_{n}^{2}$ for $n \geq 4$. Indeed, if $x_{1}=0$ and $x_{j} \neq 0$ for some $j \in 2, \ldots, n$ then $f(x)<0$. But for any choice of real symmetric matrices $A_{i}$ we may find $x \in \mathbb{R}^{n} \backslash\{0\}$ with $x_{1}=0$ such that the first row of $x_{1} \mathbb{I}+x_{2} A_{2}+\cdots+x_{n} A_{n}$ is zero. Cf. [23, p.2498].

7. Singular values. Let $A$ be any $m \times n$ matrix with complex entries and let

$$
\sigma_{1}^{\downarrow}(A) \geq \sigma_{2}^{\downarrow}(A) \geq \cdots \geq \sigma_{n}^{\downarrow}(A) \geq 0
$$

be the singular values of $A$ in decreasing order, i.e., the non-negative square roots of the eigenvalues of $A^{*} A$. If $\operatorname{rank} A=\ell$, then $\sigma_{\ell+1}^{\downarrow}(A)=\cdots=\sigma_{n}^{\downarrow}(A)=0$. Thus we set $\ell:=\min \{m, n\}$ and consider only $\sigma_{j}^{\downarrow}(A)$, for $j=1, \ldots, \ell$.

We may consider the $\sigma_{j}^{\downarrow}$ as functions on the vector space $\mathbb{C}^{m \times n}$ of complex $m \times n$ matrices. Without loss of generality assume that $m \leq n$ and let $\tilde{A}$ be the 
$n \times n$ matrix resulting from $A$ by adding $n-m$ rows consisting of zeros. Then the eigenvalues of the Hermitian matrix

$$
\left(\begin{array}{cc}
0 & \tilde{A} \\
\tilde{A}^{*} & 0
\end{array}\right)
$$

are precisely

$$
\sigma_{1}^{\downarrow}(A) \geq \cdots \geq \sigma_{n}^{\downarrow}(A) \geq-\sigma_{n}^{\downarrow}(A) \geq \cdots \geq-\sigma_{1}^{\downarrow}(A) .
$$

It follows from Corollary 6.1 that, for all $k=1, \ldots, \ell$, the sum

$$
\sum_{j=1}^{k} \sigma_{j}^{\downarrow}
$$

of the $k$ largest singular values is a sublinear function on $\mathbb{C}^{m \times n}$ viewed as a real vector space. In fact, the sums $A \mapsto \sum_{j=1}^{k} \sigma_{j}^{\downarrow}(A)$ are the so-called Ky Fan norms. We obtain

Corollary 7.1. The mapping $\sigma^{\downarrow}=\left(\sigma_{1}^{\downarrow}, \ldots, \sigma_{\ell}^{\downarrow}\right): \mathbb{C}^{m \times n} \rightarrow \mathbb{R}^{\ell}$, where $\ell=$ $\min \{m, n\}$, is globally Lipschitz and difference-convex on $\mathbb{C}^{m \times n}$.

The following theorem is a consequence of Theorem 6.2

Theorem 7.2. Let $A: \mathbb{R} \rightarrow \mathbb{C}^{m \times n}$ be a curve of $m \times n$ complex matrices of class $C^{1} \cap D C \cap W_{\text {loc }}^{2,1}$. If $\operatorname{rank} A(t)=\min \{m, n\}=: \ell$ for all $t$, then there exists a system $\sigma=\left(\sigma_{1}, \ldots, \sigma_{\ell}\right)$ of the singular values of $A$ such that $\sigma \in C^{1}\left(\mathbb{R}, \mathbb{R}^{\ell}\right) \cap D C\left(\mathbb{R}, \mathbb{R}^{\ell}\right) \cap$ $W_{\mathrm{loc}}^{2,1}\left(\mathbb{R}, \mathbb{R}^{\ell}\right)$. This result is uniform in the sense explained in Theorem 6.2.

Proof. The assertions follow from Theorem 6.2 applied to the Hermitian matrix (7.1). The condition on the rank of $A$ guarantees that the non-trivial singular values of $A$ are always strictly positive and hence there exists a $C^{1}$-system of them, since there exists a $C^{1}$-system of the eigenvalues of (7.1).

The rank condition is necessary; for instance, the singular value of the symmetric $1 \times 1$ matrix $A(t)=(t)$ is $|t|$ which does not admit a $C^{1}$ parameterization.

\section{REFERENCES}

[1] D. Alekseevsky, A. Kriegl, M. Losik, and P. W. Michor, Choosing roots of polynomials smoothly, Israel J. Math. 105 (1998), 203-233.

[2] L. Ambrosio, N. Fusco, and D. Pallara, Functions of bounded variation and free discontinuity problems, Oxford Mathematical Monographs, The Clarendon Press Oxford University Press, New York, 2000.

[3] M. F. Atiyah, R. Bott, and L. Gärding, Lacunas for hyperbolic differential operators with constant coefficients. I, Acta Math. 124 (1970), 109-189.

[4] M. Bačák and J. M. Borwein, On difference convexity of locally Lipschitz functions, Optimization 60 (2011), no. 8-9, 961-978.

[5] H. H. Bauschke, O. Güler, A. S. Lewis, and H. S. Sendov, Hyperbolic polynomials and convex analysis, Canad. J. Math. 53 (2001), no. 3, 470-488.

[6] J.-M. Bony, F. Broglia, F. Colombini, and L. Pernazza, Nonnegative functions as squares or sums of squares, J. Funct. Anal. 232 (2006), no. 1, 137-147.

[7] J. Borcea and P. Brändén, Multivariate Pólya-Schur classification problems in the Weyl algebra, Proceedings of the London Mathematical Society 101 (2010), no. 1, 73-104.

[8] G. Bourdaud, Le calcul fonctionnel dans les espaces de Sobolev, Invent. Math. 104 (1991), no. $2,435-446$.

[9] M. D. Bronshtein, Smoothness of roots of polynomials depending on parameters, Sibirsk. Mat. Zh. 20 (1979), no. 3, 493-501, 690, English transl. in Siberian Math. J. 20 (1980), 347-352. 
[10] F. Colombini, N. Orrù, and L. Pernazza, On the regularity of the roots of hyperbolic polynomials, Israel J. Math. 191 (2012), 923-944.

[11] R. M. Dudley, On second derivatives of convex functions, Math. Scand. 41 (1977), no. 1, 159-174.

[12] L. Gårding, Linear hyperbolic partial differential equations with constant coefficients, Acta Math. 85 (1951), 1-62.

[13] _ An inequality for hyperbolic polynomials, J. Math. Mech. 8 (1959), 957-965.

[14] L. Gurvits, Van der Waerden/Schrijver-Valiant like conjectures and stable (aka hyperbolic) homogeneous polynomials: one theorem for all, Electron. J. Combin. 15 (2008), no. 1, Research Paper 66, 26, With a corrigendum.

[15] P. Hartman, On functions representable as a difference of convex functions, Pacific J. Math. 9 (1959), no. 3, 707-713.

[16] F. R. Harvey and H. B. Lawson, Jr., Gårding's theory of hyperbolic polynomials, Comm. Pure Appl. Math. 66 (2013), no. 7, 1102-1128.

[17] J. W. Helton and V. Vinnikov, Linear matrix inequality representation of sets, Commun. Pure Appl. Math. 60 (2007), no. 5, 654-674 (English).

[18] J.-B. Hiriart-Urruty, Generalized differentiability, duality and optimization for problems dealing with differences of convex functions, Convexity and duality in optimization (Groningen, 1984), Lecture Notes in Econom. and Math. Systems, vol. 256, Springer, Berlin, 1985, pp. 3770.

[19] A. Kriegl and P. W. Michor, Differentiable perturbation of unbounded operators, Math. Ann. 327 (2003), no. 1, 191-201.

[20] K. Kurdyka and L. Paunescu, Hyperbolic polynomials and multiparameter real-analytic perturbation theory, Duke Math. J. 141 (2008), no. 1, 123-149.

[21] P. D. Lax, Differential equations, difference equations and matrix theory, Comm. Pure Appl. Math. 11 (1958), 175-194.

[22] G. Leoni, A first course in Sobolev spaces, Graduate Studies in Mathematics, vol. 105, American Mathematical Society, Providence, RI, 2009.

[23] A. S. Lewis, P. A. Parrilo, and M. V. Ramana, The Lax conjecture is true., Proc. Am. Math. Soc. 133 (2005), no. 9, 2495-2499 (English).

[24] A. W. Marcus, D. A. Spielman, and N. Srivastava, Interlacing families II: Mixed characteristic polynomials and the Kadison-Singer problem, Ann. of Math. (2) 182 (2015), no. 1, 327-350.

[25] W. Nuij, A note on hyperbolic polynomials, Math. Scand. 23 (1968), 69-72 (1969).

[26] A. Parusiński and A. Rainer, A new proof of Bronshtein's theorem, J. Hyperbolic Differ. Equ. 12 (2015), no. 4, 671-688.

[27] A. Rainer, Quasianalytic multiparameter perturbation of polynomials and normal matrices, Trans. Amer. Math. Soc. 363 (2011), no. 9, 4945-4977.

[28] _ Perturbation theory for normal operators, Trans. Amer. Math. Soc. 365 (2013), no. $10,5545-5577$.

[29] F. Rellich, Störungstheorie der Spektralzerlegung, Math. Ann. 113 (1937), no. 1, 600-619.

[30] _ Perturbation theory of eigenvalue problems, Assisted by J. Berkowitz. With a preface by Jacob T. Schwartz, Gordon and Breach Science Publishers, New York, 1969.

[31] R. T. Rockafellar, Convex analysis, Princeton Mathematical Series, No. 28, Princeton University Press, Princeton, N.J., 1970.

[32] L. Veselý, J. Duda, and L. Zajíček, On d.c. functions and mappings., Atti Semin. Mat. Fis. Univ. Modena 51 (2003), no. 1, 111-138 (English).

[33] L. Veselý and L. Zajíček, Delta-convex mappings between Banach spaces and applications, Dissertationes Math. (Rozprawy Mat.) 289 (1989), 52.

[34] V. Vinnikov, Self-adjoint determinantal representations of real plane curves, Math. Ann. 296 (1993), no. 1, 453-479.

FakUltät für Mathematik, Universität Wien, Oskar-Morgenstern-Platz 1, A-1090 Wien, Austria

Email address: armin.rainer@univie.ac.at 